\title{
On the chromatic number of intersection graphs of convex sets in the plane*
}

\author{
Seog-Jin Kim \\ Department of Mathematics \\ University of Illinois, Urbana, IL 61801, USA \\ skim12@math.uiuc.edu \\ Alexandr Kostochka \\ Department of Mathematics \\ University of Illinois, Urbana, IL 61801, USA \\ and \\ Institute of Mathematics, 630090 Novosibirsk, Russia \\ kostochk@math . uiuc .edu \\ Kittikorn Nakprasit \\ Department of Mathematics \\ University of Illinois, Urbana, IL 61801, USA \\ nakprasi@math.uiuc.edu
}

Submitted: Dec 10, 2002; Accepted: May 21, 2004; Published: Aug 19, 2004

MR Subject Classifications: 05C15, 05C35

\begin{abstract}
Let $G$ be the intersection graph of a finite family of convex sets obtained by translations of a fixed convex set in the plane. We show that every such graph with clique number $k$ is $(3 k-3)$-degenerate. This bound is sharp. As a consequence, we derive that $G$ is $(3 k-2)$-colorable. We show also that the chromatic number of every intersection graph $H$ of a family of homothetic copies of a fixed convex set in the plane with clique number $k$ is at most $6 k-6$.
\end{abstract}

\section{Introduction}

The intersection graph $G$ of a family $\mathcal{F}$ of sets is the graph with vertex set $\mathcal{F}$ where two members of $\mathcal{F}$ are adjacent if and only if they have common elements. Asplund and Grünbaum [3] and Gyárfás and Lehel [11,9] started studying many interesting problems

\footnotetext{
${ }^{*}$ This work was partially supported by the NSF grants DMS-0099608 and DMS-00400498.
} 
on the chromatic number of intersection graphs of convex figures in the plane. Many problems of this type can be stated as follows. For a class $\mathcal{G}$ of intersection graphs and for a positive integer $k$, find or bound $f(\mathcal{G}, k)$ - the maximum chromatic number of a graph in $\mathcal{G}$ with the clique number at most $k$. A number of results on the topic can be found in $[5,9,11,13]$.

Recently, several papers on intersection graphs of translations of a plane figure appeared. Akiyama, Hosono, and Urabe [2] considered $f(\mathcal{C}, k)$, where $\mathcal{C}$ is the family of intersection graphs of unit squares on the plane with sides parallel to the axes. They proved that $f(\mathcal{C}, 2)=3$ and asked about $f(\mathcal{C}, k)$ and, more generally, about chromatic number of intersection graphs of unit cubes in $\mathbb{R}^{d}$. In connection with channel assignment problem in broadcast networks, Clark, Colbourn, and Johnson [4] and Gräf, Stumpf, and Weißenfels [6] considered colorings of graphs in the class $\mathcal{U}$ of intersection graphs of unit disks in the plane. They proved that finding chromatic number of graphs in $\mathcal{U}$ is an $N P$-complete problem. In $[6,18]$, and [17] polynomial algorithms are given implying that $f(\mathcal{U}, k) \leq 3 k-2$. Perepelitsa [18] also considered the more general family $\mathcal{T}$ of intersection graphs of translations of a fixed compact convex figure in the plane. She proved that every graph in $\mathcal{T}$ is $(8 k-8)$-degenerate, which implies that $f(\mathcal{T}, k) \leq 8 k-7$. She also considered intersection graphs of translations of triangles and boxes in the plane.

Recall that a graph $G$ is called $m$-degenerate if every subgraph $H$ of $G$ has a vertex $v$ of degree at most $m$ in $H$. It is well known that every $m$-degenerate graph is $(m+1)$ colorable. In fact, the property of being $m$-degenerate is sufficiently stronger that being $(m+1)$-colorable. In particular, every $m$-degenerate is also $(m+1)$-list-colorable.

Our main result strengthens Perepelitsa's bound as follows.

Theorem 1 Let $G$ be the intersection graph of translations of a fixed compact convex set in the plane with clique number $\omega(G)=k$. Then $G$ is $(3 k-3)$-degenerate. In particular, the chromatic number and the list chromatic number of $G$ do not exceed $3 k-2$.

The bound on degeneracy in Theorem 1 is sharp. In Section 5 , for every $k \geq 2$ we present the intersection graph $G$ of a family of unit circles in the plane with $\omega(G)=k$ that is not $(3 k-4)$-degenerate.

The idea of the proof of Theorem 1 allows us to estimate the maximum degree of the intersection graph.

Theorem 2 Let $G$ be the intersection graph of translations of a fixed compact convex set in the plane with $\omega(G)=k, k \geq 2$. Then the maximum degree of $G$ is at most $6 k-7$.

This bound is also sharp.

Then we consider a more general setting: shrinking and blowing of the figures are now allowed.

Theorem 3 Let $H$ be the intersection graph of a family $F$ of homothetic copies of a fixed convex compact set $D$ in the plane. If $\omega(H)=k, k \geq 2$, then $H$ is $(6 k-7)$-degenerate. In particular, the chromatic number and the list chromatic number of $H$ do not exceed $6 k-6$. 
There is no upper bound on the maximum degree for intersection graphs of homothetic copies of a fixed convex set in the plane analogous to Theorem 2 , since every star is a graph of this type.

The results above yield some Ramsey-type bounds for geometric intersection graphs. For a positive integer $n$ and a family $\mathcal{F}$ of graphs, let $r(\mathcal{F}, n)$ denote the maximum $r$ such that for every $G \in \mathcal{F}$ on $n$ vertices, either the clique number, $\omega(G)$, or the independence number, $\alpha(G)$, is at least $r$. One can read Ramsey Theorem for graphs as the statement that for the family $\mathcal{G}$ of all graphs, $r(\mathcal{G}, n) \sim 0.5 \log _{2} n$. Larman, Matousek, Pach, and Torocsik [15] proved that for the family $\mathcal{P}$ of intersection graphs of compact convex sets in the plane, $r(\mathcal{P}, n) \geq n^{0.2}$. Since $\chi(H) \geq \frac{n}{\alpha(H)}$ for every $n$-vertex graph $H$, Theorem 3 yields that for every $n$-vertex intersection graph $H$ of a family of homothetic copies of a fixed convex compact set $D$ in the plane, we have $\alpha(H)(6 \omega(H)-7) \geq n$. It follows that $r(\mathcal{D}, n) \geq \sqrt{n / 6}$ for the family $\mathcal{D}$ of intersection graphs of homothetic copies of a fixed convex compact set in the plane. Similarly, Theorem 1 yields that $r(\mathcal{T}, n) \geq \sqrt{n / 3}$.

The structure of the paper is as follows. In the next section we introduce our tools. Theorems 1 and 2 are proved in Section 3. In Section 4 we prove Theorem 3. Section 5 is devoted to construction of extremal graphs.

\section{Preliminaries}

Given sets $A$ and $B$ of vectors and a real $\alpha$, the set $\alpha(A+B)$ is defined as $\{\alpha(a+b) \mid a \in$ $A, b \in B\}$. When $B=\{b\}$, we sometimes write $A+b$ instead of $A+\{b\}$. Our first tool is the following lemma.

Lemma 4 Let $A$ be a convex figure and $A^{\prime \prime}=A+s$ where $s$ is a vector. Let $P$ be a convex figure that intersects both $A$ and $A^{\prime \prime}$. If $A^{\prime}=A+\alpha s, 0 \leq \alpha \leq 1$, then $P$ intersects $A^{\prime}$.

Proof. Let $u \in A \cap P, u^{\prime \prime}=u+s, v^{\prime \prime} \in A^{\prime \prime} \cap P, v=v^{\prime \prime}-s$. So $v^{\prime \prime} \in A^{\prime \prime}, u \in A$, and the interval $\overline{u v^{\prime \prime}}$ is in $P$. Let $A^{\prime}=A+\alpha s, u^{\prime}=u+\alpha s$, and $v^{\prime}=v+\alpha s$. Then the interval $\overline{u^{\prime} v^{\prime}}$ is in $A^{\prime}$ and must intersect the interval $\overline{u v^{\prime \prime}}$ in $P$.

Our second tool is an old result of Minkowski [16].

Lemma 5 (Minkowski [16]) Let $K$ be a convex set in the plane. Then $(x+K) \cap(y+K) \neq$ $\emptyset$ if and only if $\left(x+\frac{1}{2}[K+(-K)]\right) \cap\left(y+\frac{1}{2}[K+(-K)]\right) \neq \emptyset$.

A proof can be also found in [12]. Note that the set $\frac{1}{2}[K+(-K)]$ is centrally symmetric for every $K$. Hence without loss of generality, it is enough to prove Theorems 1, 2, and 3 for centrally symmetric convex sets. For handling these sets, the notion of Minkowski norm is quite useful.

Let $K$ be a compact convex set on the plane, centrally symmetric about the origin. For every point $x$ on the plane, we define the Minkowski norm 


$$
\|x\|_{K}=\inf _{\lambda \geq 0}\{\lambda \in \mathbb{R}: x \in \lambda K\}
$$

Note that $\left\{x:\|x\|_{K}=1\right\}$ is the boundary of $K$. It is easily checked that $u+K$ and $v+K$ intersect if and only if $\|u-v\|_{K} \leq 2$. The two lemmas below appear in [8]. We present their proofs, since they are very short.

Lemma 6 (Grünbaum [8]) Let $x, y, z$ be different points belonging to the boundary of $K$, such that the origin $O$ does not belong to the open half-plane determined by $x$ and $y$ that contains $z$. Then $\|x-z\|_{K} \leq\|x-y\|_{K}$.

Proof. If $x+y=0$, then $\|x-y\|_{K}=\|2 x\|_{K}=2$, since $x$ is on the boundary of $K$. On the other hand, $\|x-z\|_{K} \leq\|x\|_{K}+\|z\|_{K}=2$. Hence $\|x-z\|_{K} \leq\|x-y\|_{K}$.

If $x+y \neq 0$, find another triple of points $x^{*}, y^{*}, z^{*}$ such that $x^{*}+y^{*}=0$ and the triangle with vertices $\left\{x^{*}, y^{*}, z^{*}\right\}$ is similar to $\{x, y, z\}$. We can check that $z^{*}$ is inside $K$, hence $\left\|x^{*}-z^{*}\right\|_{K} \leq\left\|x^{*}-y^{*}\right\|_{K}$. Therefore $\|x-z\|_{K} \leq\|x-y\|_{K}$.

Lemma 7 (Grünbaum [8]) Let $x, y, z, u$ be different points belonging to the boundary of $K$, such that $z$ and $u$ belong to an open half-plane determined by $x$ and $y$, while $O$ belongs to its complement. Then $\|z-u\|_{K} \leq\|x-y\|_{K}$.

Proof. We may assume that the points are located in order $x, u, z, y$ counterclockwise. From Lemma $6,\|z-u\|_{K} \leq\|x-z\|_{K} \leq\|x-y\|_{K}$.

\section{Proofs of Theorems 1 and 2}

It will be convenient to prove the following slightly refined version of Theorem 1 for centrally symmetric sets.

Theorem 8 Let $\mathcal{M}=\left\{M_{i}\right\}$ be a set of translates of a centrally symmetric convex set in the plane with given axes. If the clique number of the intersection graph $G(\mathcal{M})$ of $\mathcal{M}$ is $k$, then every highest member $A$ of $\mathcal{M}$ intersects at most $3 k-3$ other members.

Proof. For an arbitrary set $S$, define $\mathcal{M}(S)=\left\{M_{i} \in \mathcal{M} \mid S \cap M_{i} \neq \emptyset\right\}$. Let $A$ be a highest member of $\mathcal{M}$. For convenience, we assume that the center of $A$ is the origin $O=(0,0)$. Let $z$ be the rightmost point on the $X$-axis that belongs to $A$. If $z=(0,0)$, then $A$ is an interval with the center $O$ and $G$ is an interval graph. So, we assume $z \neq(0,0)$. Let $B=A-2 z$ and $C=A+2 z$. Since $A$ is convex and centrally symmetric, $B$ and $C$ touch $A$ but have no common interior points with $A$. Note that $B$ and $C$ may or may not belong to $\mathcal{M}$.

The following three claims are crucial for our proof.

Claim 3.1 Let $\mathcal{M}_{1}(A)=\mathcal{M}(A) \cap \mathcal{M}(B)$. Then every two members of $\mathcal{M}_{1}(A)$ intersect. 
Claim 3.2 Let $\mathcal{M}_{2}(A)=\mathcal{M}(A) \cap \mathcal{M}(C)$. Then every two members of $\mathcal{M}_{2}(A)$ intersect.

Claim 3.3 Let $\mathcal{M}_{3}(A)=\mathcal{M}(A)-\mathcal{M}(B)-\mathcal{M}(C)$. Then every two members of $\mathcal{M}_{3}(A)$ intersect.

Indeed, $\mathcal{M}(A)=\mathcal{M}_{1}(A) \cup \mathcal{M}_{2}(A) \cup \mathcal{M}_{3}(A)$. If Claims 3.1, 3.2, and 3.3 hold, then, since $\omega(G)=k,\left|\mathcal{M}_{i}(A)\right| \leq k-1$ for $i=1,2,3$, and hence $A$ intersects at most $3 k-3$ members of $\mathcal{M}$. Therefore, we need only to prove the claims.

Let $L$ be a supporting line for $A$ at $(-z, 0)$, i.e. a line passing through $(-z, 0)$ and having no common points with the interior of $A$. Such a line exists, since $A$ is convex. If $(-z, 0)$ is a corner of $A$, then $L$ is not unique. Furthermore, since $A$ is centrally symmetric, $L$ is also a supporting line for $B$. Below, we will use the (not necessary orthogonal) coordinate system with the same origin and the $X$-axis as we used above, but whose $Y$-axis is parallel to $L$. We scale the new $Y$-axis so that the new $y$-coordinate of every point is the same as the old one.

Proof of Claim 3.1. Let $L_{A}=L+z$ and $L_{B}=L-z$ be the straight lines that are parallel to $L$ and pass through the center $(0,0)$ of $A$ and the center $-2 z$ of $B$, respectively. Note that $L_{A}$ is the new $y$-axis. Let $S$ be the strip between $L_{A}$ and $L_{B}$ on the plane.

Let $U$ and $V$ be in $\mathcal{M}_{1}(A), U=u+A$ and $V=v+A, u=\left(x_{u}, y_{u}\right), v=\left(x_{v}, y_{v}\right)$. Without loss of generality, we may assume that $x_{u} \geq x_{v}$. Note that $u$ and $v$ are in the strip $S$.

Case 1. $y_{u} \geq y_{v}$. Let $L_{u}=L_{A}+u$. This line passes through $u$ and is parallel to $L$. Similarly, $L_{v}=L_{A}+v$ passes through $v$ and is parallel to $L$. Let $u^{\prime}$ (respectively, $v^{\prime}$ ) be the intersection point of the $x$-axis and $L_{u}$ (respectively, $L_{v}$ ) and $U^{\prime}=A+u^{\prime}$ (respectively, $\left.V^{\prime}=A+v^{\prime}\right)$. Since $U^{\prime}$ and $V^{\prime}$ are between $A$ and $B$, by Lemma $4, U^{\prime}$ and $V^{\prime}$ intersect $V$ and each other. Let $u^{\prime \prime}$ be the point on $L_{u}$ with the $y$-coordinate equal to $y_{v}$ and $U^{\prime \prime}=A+u^{\prime \prime}$. Since $U^{\prime}$ intersects $V^{\prime}, U^{\prime \prime}$ intersects $V$. But $U$ is located between $U^{\prime}$ and $U^{\prime \prime}$ (or coincides with $U^{\prime \prime}$ if $y_{u}=y_{v}$ ). Therefore, Lemma 4 implies that $U$ intersects $V$.

Case 2. $y_{u}<y_{v}$. Repeating the proof of Case 1 with roles of $A$ and $B$ switched yields this case.

The proof of Claim 3.2 is the same (with $C$ in place of $B$ ).

Proof of Claim 3.3. Let $A^{*}=2 A, B^{*}=A^{*}-2 z$, and $C^{*}=A^{*}+2 z$. Let $s=\left(x_{s}, y_{s}\right)$ be a lowest intersection point of $A^{*}$ and $B^{*}$. Since $A^{*}=B^{*}+2 z$ and $C^{*}=A^{*}+2 z$, the point $w=s-2 z$ belongs to $B^{*}$ and the point $t=s+2 z$ is an intersection point of $A^{*}$ and $C^{*}$. Furthermore, $\|s-t\|_{A}=\|s-w\|_{A}=2$.

Let $W$ denote the figure bounded by the straight line segment from $O$ to $s$, the arc of the boundary of $A^{*}$ from $s$ to $t$, denoted by $R_{2}$, and the straight line segment $t$ to $O$ (see Fig. 2). We will prove now that $W$ contains all the points of $A^{*}-\left(B^{*} \cup C^{*}\right)$ with non-positive $y$-coordinates. Indeed, suppose that $A^{*}-\left(B^{*} \cup C^{*}\right)$ contains a point $u=\left(x_{u}, y_{u}\right)$ with $y_{u} \leq 0$ on the left of the line $l_{s}$ passing through $O$ and $s$. Then, by the definition of $B^{*}$, the point $u^{\prime}=u-2 z$ belongs to $B^{*}$. 


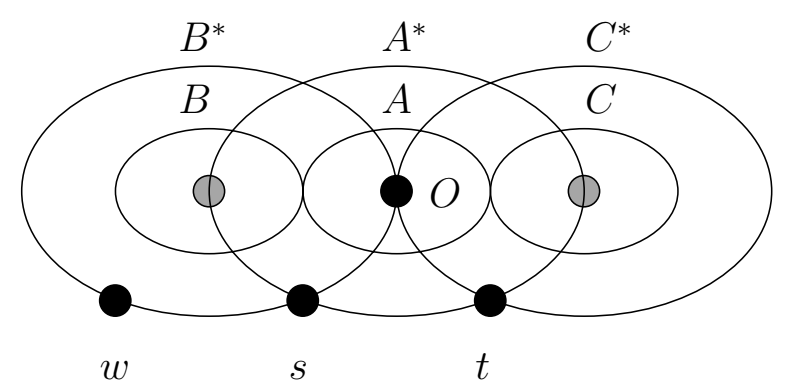

Figure 1: The intersection of boundaries of $A^{*}$ and $B^{*}$.

CASE 1. $y_{u}<y_{s}$. Then the straight line segment $I_{1}$ from $u^{\prime}$ to $s$ is contained in $B^{*}$ and the straight line segment $I_{2}$ from $u$ to $O-2 z$ is contained in $A^{*}$. Moreover, $I_{2}$ crosses $I_{1}$, and their crossing point, $u^{*}$, has the $y$-coordinate less than $s$ (since it belongs to $I_{1}$ ). But $u^{*} \in A^{*} \cap B^{*}$, a contradiction to the choice of $s$.

CASE 2. $y_{s} \leq y_{u} \leq 0$. Let $u^{\prime \prime}$ be the intersection point of $l_{s}$ and the line $y=y_{u}$. Since $u^{\prime \prime}$ is between $O$ and $s$ on $l_{s}$, it belongs to $B^{*}$. Therefore, all points on the interval between $u^{\prime}$ and $u^{\prime \prime}$ belong to $B^{*}$. In particular, $u \in B^{*}$, a contradiction to $u \in A^{*}-\left(B^{*} \cup C^{*}\right)$.

Similarly, $A^{*}-\left(B^{*} \cup C^{*}\right)$ cannot contain points with non-positive $y$-coordinates on the right of the line $l_{t}$ passing through $O$ and $t$.

Let $U, V \in \mathcal{M}_{3}(A), U=u+A, V=v+A, u=\left(x_{u}, y_{u}\right), v=\left(x_{v}, y_{v}\right)$. Then by definition, $y_{u} \leq 0$, and $y_{v} \leq 0$, and by the above, $u, v \in W$. As it was pointed out in Section 2, proving that $U$ and $V$ intersect is equivalent to proving that $\|u-v\|_{A} \leq 2$.

Let $u, v \in W$ and let $l_{u}$ (respectively, $l_{v}$ ) be the straight lines passing through $O$ and $u$ (respectively, $v$ ). Since $B^{*}$ and $C^{*}$ are convex, the lines $l_{u}$ and $l_{v}$ must pass between the straight line $l_{s}$ and the straight line $l_{t}$ (see Fig. 2). Since $R_{2}$ connects $s$ with $t$, we conclude that lines $l_{u}$ and $l_{v}$ intersect $R_{2}$. Let $u^{\prime}$ (respectively, $v^{\prime}$ ) be the intersection point of $l_{u}$ (respectively, $l_{v}$ ) and $R_{2}$. By Lemmas 6 and $7,\left\|u^{\prime}-v^{\prime}\right\|_{A} \leq\|s-t\|_{A}=\|2 z\|_{A}=2$. Hence $u^{\prime}+A$ and $v^{\prime}+A$ intersect (and both intersect $A$ ). Since $v$ is between $O$ and $v^{\prime}$ on $l_{v}$, Lemma 4 yields that $u^{\prime}+A$ intersects $v+A$. Now, since $u$ is between $O$ and $u^{\prime}$ on $l_{u}$, the same lemma yields that $v+A$ intersects $u+A$. This proves the claim and thus the theorem.

Clearly, Theorem 8 implies Theorem 1. Now we also derive Theorem 2.

Proof of Theorem 2. Let $A$ be a member of a set $\mathcal{M}=\left\{M_{i}\right\}$ of translates of a centrally symmetric convex set in the plane such that the clique number of the intersection graph $H(\mathcal{M})$ of $\mathcal{M}$ is $k$. We want to prove that $A$ intersects at most $6 k-7$ other members of $\mathcal{M}$. Let $B \in \mathcal{M}$ intersect $A$. Choose a coordinate system on the plane so that the center of $A$ is the origin and the center of $B$ lies on the $x$-axis. Let $\mathcal{M}^{+}$(respectively, $\mathcal{M}^{-}$) be the family of members of $\mathcal{M}$ with a nonnegative (respectively, non-positive) $y$-coordinate. Then $A$ is a highest member of $\mathcal{M}^{-}$and a lowest member of $\mathcal{M}^{+}$. By Theorem $8, A$ has at most $3 k-3$ neighbors in each of $\mathcal{M}^{-}$and $\mathcal{M}^{+}$. Moreover, $B$ was counted in both sets. This proves the theorem. 


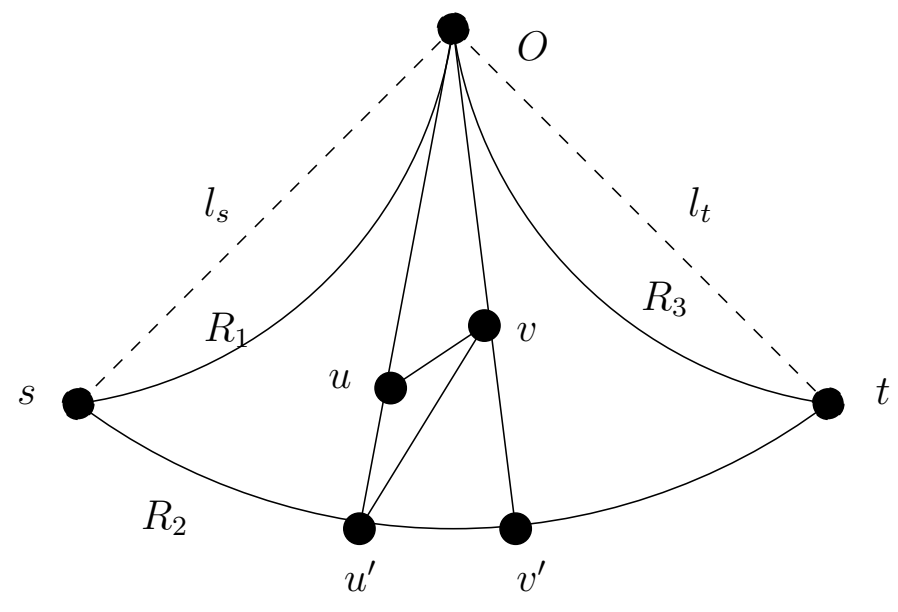

Figure 2: $\mathcal{M}_{3}(A)=\mathcal{M}(A)-\mathcal{M}(B)-\mathcal{M}(C)$

\section{Intersection graphs of convex sets with different sizes}

In this section, we study a more general case. Two convex sets $K, D$ on the plane are called homothetic if $K=x+\lambda D$ for a point $x$ on the plane and some $\lambda>0$. We consider intersection graphs of families of homothetic copies of a fixed compact convex set. Any intersection graph of a family of different sized circles is a special example. Note that Lemma 5 does not need to hold in this more general case.

The following easy observation is quite useful for our purposes.

Lemma 9 Let $U$ be a convex set containing the origin. For each $v \in U$ and $0 \leq \lambda \leq 1$, the set $W(U, v, \lambda)=(1-\lambda) v+\lambda U$ is contained in $U$ and contains $v$.

Proof. By the definition, $W(U, v, \lambda)=\{v+\lambda \cdot(u-v) \mid u \in U\}$.

Let $u \in U$. Since $v+0 \cdot(u-v)=v \in U, v+1 \cdot(u-v)=u \in U$, and $U$ is convex, we have $v+\lambda \cdot(u-v) \in U$ for every $0 \leq \lambda \leq 1$. On the other hand, $v=v+\lambda \cdot(v-v) \in W(U, v, \lambda)$ for every $0 \leq \lambda \leq 1$.

Proof of Theorem 3. Let $Z$ be a smallest homothetic copy of $D$ in $F$. Let $F(Z)$ be the set of members of $F$ intersecting $Z$. For every $U \in F(Z)$, let $\lambda(U)$ be the positive real such that $Z=u+\lambda(U) U$ for some $u$. For every $U \in F(Z)$, choose a point $z(U) \in Z \cap U$ and denote $U^{*}=W(U, z(U), \lambda(U))=(1-\lambda(U)) z(U)+\lambda(U) U$. Note that $U^{*}$ is a translate of $Z$. By Lemma 9, the intersection graph $G$ of the family $F^{*}(Z)=\{Z\} \cup\left\{U^{*} \mid U \in F(Z)\right\}$ is a subgraph of $H$. In particular, the clique number of $G$ is at most $k$. Moreover, because of the choice of $z(U), \operatorname{deg}_{G}(Z)=\operatorname{deg}_{H}(Z)$. Since $F^{*}(Z)$ consists of translates of $Z$, Theorem 2 implies that $\operatorname{deg}_{G}(Z) \leq 6 k-7$.

Remark. It is known that the maximum degree of any intersection graph of translations of a box in the plane with clique number $k$ is at most $4 k-4$. Repeating the proof 
of Theorem 3 for this special case, we obtain that every intersection graph of homothetic copies of a box in the plane with clique number $k$ is $(4 k-4)$-degenerate.

\section{Constructions}

Our first example shows that the bound on the maximum degree in Theorem 2 is sharp.

Example 1. Let $K$ be the unit circle whose center is the origin in the plane. Let $K_{2}$ be the circle of radius 2 whose center is the origin. For $0 \leq i \leq 6 k-8$, let $v_{i}$ be the point on the boundary of $K_{2}$ with the polar coordinates $\left(2, i \frac{2 \pi}{6 k-7}\right)$. Let $A_{i}=K+v_{i}$ for $0 \leq i \leq 6 k-8$. Then $K$ intersects $A_{i}$ for all $i$. Observe that $A_{i}$ intersects $A_{j}$ if and only if $|i-j| \leq k-2(\bmod 6 k-7)$. It follows that the clique number of the intersection graph $G$ of the family $\{K\} \cup\left\{A_{i}: 0 \leq i \leq 6 k-8\right\}$ is $k$ and the degree of $K$ in $G$ is $6 k-7$.

Example 2. Fix a positive real $R$. For a positive integer $m$, let $F_{m}^{\prime}=\left\{\left(R, \frac{i}{m-1 / 2}\right): i=\right.$ $0, \pm 1, \pm 2, \ldots\}, F_{m}^{\prime \prime}=\left\{\left(R-\sqrt{3}, \frac{i}{m-1 / 2}\right): i=0, \pm 1, \pm 2, \ldots\right\}$, and $F_{m}=F_{m}^{\prime} \cup F_{m}^{\prime \prime}$. In other words, we choose an infinite number of points on the vertical lines $x=R$ and $x=R-\sqrt{3}$. A part of $F_{m}^{\prime} \cup F_{m}^{\prime \prime}$ is drawn on Figure 3 (left). Let $\mathcal{C}_{m}$ be the family of unit circles in the plane with the set of centers $F_{m}^{\prime} \cup F_{m}^{\prime \prime}$ and let $G_{m}$ be the intersection graph of $\mathcal{C}_{m}$. It is convenient to view $G_{m}$ as the graph with the vertex set $F_{m}^{\prime} \cup F_{m}^{\prime \prime}$ such that two points $u$ and $v$ are adjacent if and only if the (Euclidean) distance $\rho(u, v)$ is at most 2. We derive some properties of $G_{m}$ in a series of claims.

The first claim is evident.

Claim $5.1 \rho\left(\left(R, y_{1}\right),\left(R-\sqrt{3}, y_{2}\right)\right) \leq 2$ if and only if $\left|y_{1}-y_{2}\right| \leq 1$.

This simple fact and the definition of $F_{m}$ imply the next claim.

Claim 5.2 If $\left(R, y_{1}\right) \in F_{m}^{\prime}$, then the maximum (respectively, minimum) $y_{2}$ such that $\left(R-\sqrt{3}, y_{2}\right) \in F_{m}^{\prime \prime}$ and $\rho\left(\left(R, y_{1}\right),\left(R-\sqrt{3}, y_{2}\right)\right) \leq 2$ is $y_{2}=y_{1}+1-\frac{1}{2 m-1}$ (respectively, $\left.y_{2}=y_{1}-1+\frac{1}{2 m-1}\right)$.

It follows that every $u \in F_{m}$ has $2(2 m-1)$ neighbors on the same vertical line and $2 m-1$ neighbors on the other vertical line. Thus, we have

Claim 5.3 For every $u \in F_{m}$, $\operatorname{deg}_{G_{m}}(u)=6 m-3$.

Let $Q \subset F_{m}$ be a maximum clique in $G_{m}, Q_{1}=Q \cap F_{m}^{\prime}, Q_{2}=Q \cap F_{m}^{\prime \prime}$. Suppose that the lowest point $v_{i}$ in $Q_{i}, i=1,2$, has the $y$-coordinate $y_{i}$, and the highest point $u_{i}$ in $Q_{i}$ has the $y$-coordinate $y_{i}+\frac{s_{i}}{m-1 / 2}$. Then $|Q|=s_{1}+s_{2}+2$. On the other hand, by Claim 5.2, we have

$$
y_{2} \geq y_{1}+\frac{s_{1}}{m-1 / 2}-1+\frac{1}{2 m-1} \quad \text { and } \quad y_{1} \geq y_{2}+\frac{s_{2}}{m-1 / 2}-1+\frac{1}{2 m-1} .
$$


Summing the last two inequalities we get

$$
0 \geq \frac{s_{1}+s_{2}+1}{m-1 / 2}-2
$$

i.e., $s_{1}+s_{2}+1 \leq 2 m-1$. Hence, we have

Claim $5.4 \omega\left(G_{m}\right) \leq 2 m$.

Thus, for every even $k$, the graph $G_{k / 2}$ is a $(3 k-3)$-regular intersection graph of unit circles with clique number $k$. A bad side of $G_{k / 2}$ is that it is an infinite graph. In order to obtain a finite graph with properties of $G_{k / 2}$, we first add one more observation on $G_{m}$.
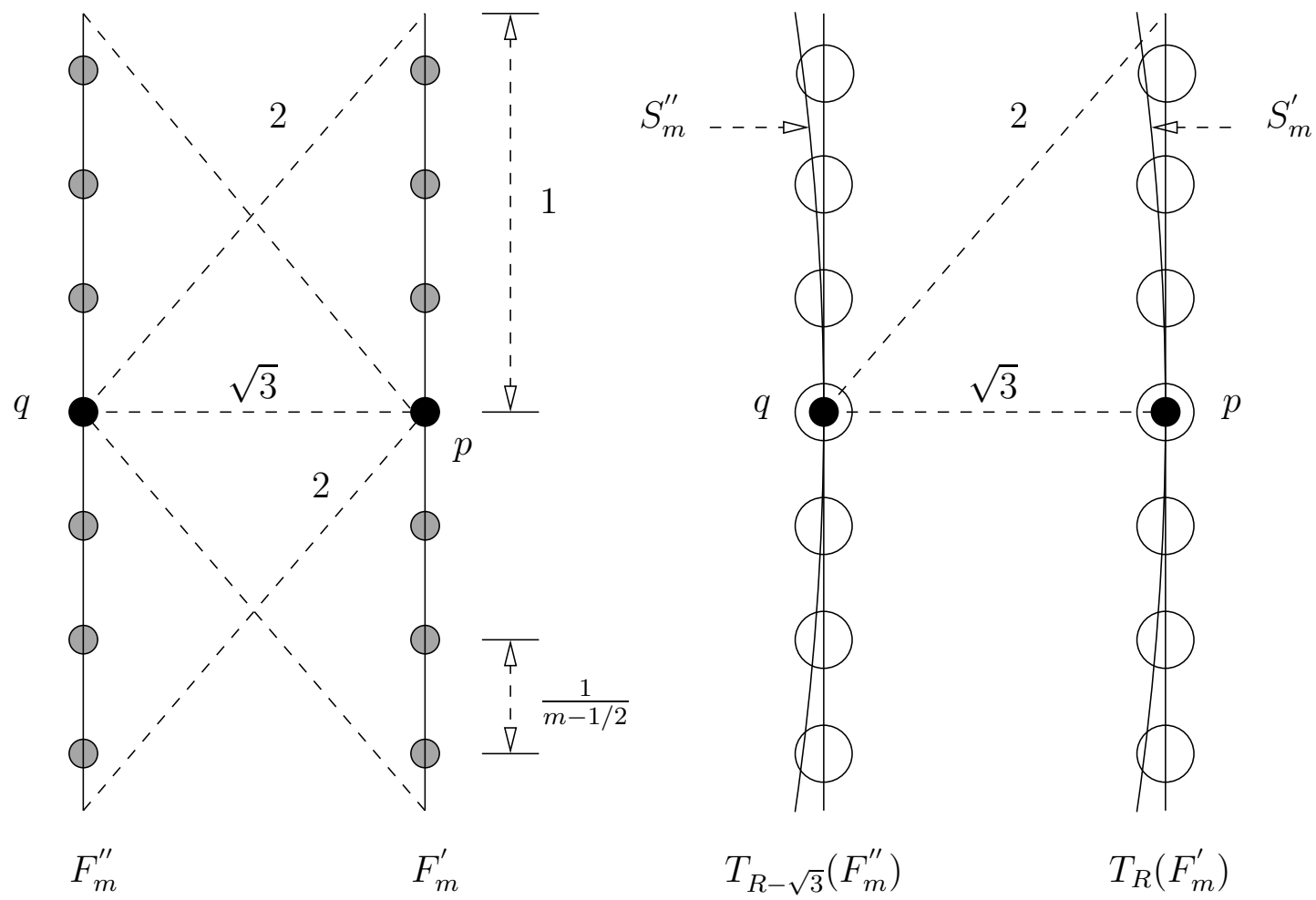

Figure 3: A fragment of $F_{4}$ (left) and $S_{4}$ (right)

Claim 5.5 Let $u \in F_{m}^{\prime}, v \in F_{m}^{\prime \prime}$. If $\rho(u, v) \leq 2$, then $\rho(u, v)<2-\frac{1}{8 m}$. If $\rho(u, v)>2$, then $\rho(u, v) \geq 2+\frac{1}{8 m}$ for $m \geq 2$.

Proof. Assume that $\rho(u, v) \leq 2$. Then by Claim 5.2,

$$
\begin{aligned}
2-\rho(u, v) & \geq 2-\sqrt{3+\left(1-\frac{1}{2 m-1}\right)^{2}}=\frac{4-3-\left(1-\frac{1}{2 m-1}\right)^{2}}{2+\sqrt{3+\left(1-\frac{1}{2 m-1}\right)^{2}}} \\
& \geq \frac{1}{4}\left(\frac{2}{2 m-1}-\frac{1}{(2 m-1)^{2}}\right) \geq \frac{1}{4}\left(\frac{1}{2 m-1}\right)>\frac{1}{8 m} .
\end{aligned}
$$


The calculations for the second inequality are very similar.

Now, let $N$ be a big positive integer (say, $N=10^{6}$ ) and $R=\frac{(2 m-1) N}{\pi}$. Consider the transformation $T$ of the plane moving every point with Cartesian coordinates $(x, y)$ into the point with polar coordinates $\left(|x|, \frac{y}{R}\right)$. For every positive $x_{0}$, the function $T_{x_{0}}(y)=T\left(x_{0}, y\right)$ is a periodic function with period $\pi R=(2 m-1) N$ mapping the line $x=x_{0}$ onto the circle $x^{2}+y^{2}=x_{0}^{2}$. Let $S_{m}^{\prime}=T\left(F_{m}^{\prime}\right)=T_{R}\left(F_{m}^{\prime}\right)$ and $S_{m}^{\prime \prime}=T\left(F_{m}^{\prime \prime}\right)=T_{R-\sqrt{3}}\left(F_{m}^{\prime \prime}\right)$. Then

$S_{m}^{\prime}=\left\{\left(R \cos \frac{2 \pi j}{(2 m-1)^{2} N}, R \sin \frac{2 \pi j}{(2 m-1)^{2} N}\right): j=0,1, \ldots,(2 m-1)^{2} N\right\}$ and

$S_{m}^{\prime \prime}=\left\{\left((R-\sqrt{3}) \cos \frac{2 \pi j}{(2 m-1)^{2} N},(R-\sqrt{3}) \sin \frac{2 \pi j}{(2 m-1)^{2} N}\right): j=0, \ldots,(2 m-1)^{2} N\right\}$.

We claim that the intersection graph $H_{m}$ of unit circles with centers in $S_{m}=S_{m}^{\prime} \cup S_{m}^{\prime \prime}$ is also $(6 m-3)$-regular and has clique number $2 m$. The reason for this is that if two points in $F_{m}$ are 'far' (i.e., on distance more than 2) and the corresponding points in $S_{m}$ do not coincide, then these corresponding points also are 'far' apart, and that if two points in $F_{m}$ are 'close', then the distance between them in $S_{m}$ is almost the same. It is enough to consider situations with points $p=(R, 0)$ and $q=(R-\sqrt{3}, 0)$ (see Fig.3 (right)). Recall that $T(p)=p$ and $T(q)=q$.

Let $B$ be the box $\{(x, y): R-\sqrt{3} \leq x \leq R ;-3 \leq y \leq 3\}$. We want to prove that for every point $u \in B \cap F_{m}$, the distance from $u$ to $p$ (respectively, $q$ ) is at most 2 if and only if the distance from $T(u)$ to $p$ (respectively, $q$ ) is at most 2 . Let $s=\left(x_{0}, y_{0}\right)$ be a point in $B$. Then $T(s)=\left(x_{0} \cos \frac{y_{0}}{R}, x_{0} \sin \frac{y_{0}}{R}\right)$. Observe that

$$
x_{0}-x_{0} \cos \frac{y_{0}}{R}=2 x_{0} \sin ^{2} \frac{y_{0}}{2 R} \leq 2 R\left(\frac{3}{2 R}\right)^{2} \leq \frac{9}{2 R}<\frac{1}{20 m} .
$$

Similarly, $y_{0}-x_{0} \sin \frac{y_{0}}{R}=\left(y_{0}-x_{0} \frac{y_{0}}{R}\right)+x_{0}\left(\frac{y_{0}}{R}-\sin \frac{y_{0}}{R}\right)$,

$$
\begin{gathered}
\left|y_{0}-x_{0} \frac{y_{0}}{R}\right| \leq \frac{\left|y_{0}\right|(R-R+2)}{R} \leq \frac{6}{R}<\frac{1}{40 m} \text { and } \\
\left|x_{0}\left(\frac{y_{0}}{R}-\sin \frac{y_{0}}{R}\right)\right| \leq x_{0}\left|\left(\frac{y_{0}}{R}\right)^{3} / 6\right| \leq R \frac{27}{6 R^{3}}<\frac{1}{40 m} .
\end{gathered}
$$

Therefore, for every $b \in B$, the distance between $b$ and $T(b)$ is less than $\frac{1}{10 m}$.

Let $q=(R-\sqrt{3}, 0)$. For each $b \in B \cap F_{m}-p$, the distance from $q$ to $T(b)$ is less than the distance from $q$ to $b$. Hence the degree of $q$ in $H_{m}$ is at least as big as in $G_{m}$. On the other hand, since the distance between $b$ and $T(b)$ is less than $\frac{1}{10 m}$, Claim 5.5 yields that $q$ gets in $H_{m}$ no new neighbor from $B \cap F_{m}$.

The case for $p=(R, 0)$ is very similar. $T$ moves the points in $B \cap F_{m}^{\prime \prime}$ slightly away from $p$, but Claim 5.5 helps us again.

For every even $k$, this gives a finite graph $H_{k / 2}$ that is a $(3 k-3)$-regular intersection graph of unit circles with clique number $k$. 
Example 3. Fix a positive real $R$. For a positive integer $m$, let $M_{m}^{\prime}=\left\{\left(R, \frac{i+1 / 2}{m}\right)\right.$ : $i=0, \pm 1, \pm 2, \ldots\}, M_{m}^{\prime \prime}=\left\{\left(R-\sqrt{3}, \frac{i}{m}\right): i=0, \pm 1, \pm 2, \ldots\right\}$, and $M_{m}=M_{m}^{\prime} \cup M_{m}^{\prime \prime}$. This family is similar to $F_{m}$ in Example 2, but the denominator for the $y$-coordinates of points is different and points in $M_{m}^{\prime}$ are shifted by $\frac{1}{2 m}$ with respect to points in $M_{m}^{\prime \prime}$.

Essentially repeating the argument of Example 2, we can see that the clique number of the intersection graph $G_{m}^{\prime}$ of unit circles with centers in $M_{m}$ is $2 m+1$ and that $G_{m}^{\prime}$ is $6 m$-regular. Then exactly as in Example 2, we obtain from $G_{m}^{\prime}$ a finite $6 m$-regular intersection graph of unit circles in the plane with clique number $2 m+1$. This shows that the bound of Theorem 1 is tight.

Remark. We don't know whether the bound of Theorem 3 is tight or not.

\section{References}

[1] P.K. Agarwal and J. Pach, Combinatorial Geometry, John Wiley \& Sons. Inc., 1995.

[2] J. Akiyama, K. Hosono, and M. Urabe, Some combinatorial problems, Discrete Math. 116 (1993) 291-298.

[3] E. Asplund and B. Grünbaum, On a coloring problem, Math. Scand. 8 (1996) 181188.

[4] B. N. Clark, C. J. Colbourn, and D. S. Johnson, Unit disk graphs, Discrete Math. 86 (1990), 165-177.

[5] M. Golumbic, Algorithmic graph theory and perfect graphs. Academic Press, 1980.

[6] A. Gräf, M. Stumpf, and G. Weißenfels, On Coloring Unit Disk Graphs, Algorithmica 20 (1998) 277-293.

[7] B. Grünbaum, On a Conjecture of H. Hadwiger, Bull. Res. Counc. of Israel, 7F (1957), 215-219.

[8] B. Grünbaum, Borsuk's Partition Conjecture in Minkowski Planes, Bull. Res. Counc. of Israel, 7F, (1957), 25-30.

[9] A. Gyárfás, Problems from the world surrounding perfect graphs. Proceedings of the International Conference on Combinatorial Analysis and its Applications (Pokrzywna, 1985). Zastos. Mat. 19 (1987), 413-441 (1988).

[10] A. Gyárfás, On the chromatic number of multiple interval graphs and overlap graphs, Discrete Math. 55 (1985), 161-166.

[11] A. Gyárfás, J. Lehel, Covering and coloring problems for relatives of intervals, Discrete Math. 55 (1985), 167-180. 
[12] H. Hadwiger and H. Debrunner, Combinatorial geometry in the plane, Holt, Rinehart and Winston, New York, 1964.

[13] A. Kostochka, On upper bounds on the chromatic numbers of graphs, Transactions of the Institute of Mathematics (Siberian Branch of the Academy of Sciences of USSR), 10 (1988), 204-226 (in Russian).

[14] A.V. Kostochka and I.G. Perepelitsa, Coloring intersection graphs of boxes on the plane, Discrete Math. 220 (2000) 243-249.

[15] D. Larman, J. Matousek, J. Pach, and J. Torocsik, A Ramsey-Type Result for Convex Sets, Bull. London Math. Soc. 26 (1994) 132-136.

[16] H. Minkowski, Dichteste gitterformige Lagerung kongruenter Körper, Nachr. der K. es. der Wiss. zu Göttingen, Math. Phys. Kl., (1904), 311-355.

[17] R. Peeters, On coloring $j$-unit sphere graphs, FEW 512, Department of Economics, Tilburg University, 1991.

[18] I.G. Perepelitsa, Bounds on the chromatic number of intersection graphs of sets in the plane, Discrete Math., 262 (2003) 221-227. 